\title{
Città, personaggi e atteggiamenti verso il dialetto nel teatro di Eduardo De Filippo
}

\author{
Nicola De Blasi
}

Università di Napoli «Federico II»

\begin{abstract}
In una prospettiva tradizionale e poco convicente il teatro di Eduardo De Filippo dal punto di vista linguistico è ritenuto espressione compatta e uniforme di ambienti familiari napoletani della piccola borghesia. Tale impostazione comporta spesso l'implicita (o sottaciuta) svalutazione delle scelte linguistiche dell'autore, velatamente sospettato di non dare spazio all'autentico e popolare dialetto napoletano (a esclusione di pochi casi interpretati spesso come frutto di scelta espressiva). Con questo intervento si punta invece a dimostarre che nelle opere dell'Autore risaltano sia una variazione linguistica diastratica, connessa a diversi ambienti e luoghi urbani, sia una diversità di atteggiamenti dei personaggi verso il dialetto. Tale variazione appare credibile e verosimile rappresentazione di una realtà linguistica cittadina che da sempre, nonostante le semplificazioni e le banalizzazioni correnti (che tendono a ritenere che a Napoli si parli solo il dialetto e solo un tipo specifico di dialetto), è sempre stata molto variegata al suo interno e tutt'altro che uniforme.
\end{abstract}

Parole chiave: Eduardo De Filippo, teatro italiano, uso teatrale dei dialetti.

\begin{abstract}
From a traditional yet sadly unconvincing perspective, the theatre of Eduardo De Filippo from a linguistic point of view is regarded is compact expression and consistent with Neapolitan family environments of the lower middle classes. A basis such as this often involves implicit (or underhand) debasement of the linguistic choice of the author, covertly suspect of not giving space to the authentic and popular Neapolitan dialect (with the exclusion of a very few cases often interpreted as the fruit of expressive choice). With this intervention the focus is to demonstrate that the works of the Author are both a diatopic linguistic variation that is connected to diverse urban places and environments, and a true diversity of identification of characters with the dialect. This variation affords credible and ostensible representation of a city linguistic reality that has always been extremely variegated from within and everything else it embodies, notwithstanding the current simplifications and banalisations (that propagate the idea that they only speak the dialect and a specific type of it in Naples).
\end{abstract}

Key words: Eduardo De Filippo, Italian theatre, theatrical use of dialects. 
Le commedie della Cantata dei giorni pari e della Cantata dei giorni dispari ${ }^{1}$ permettono di seguire una variazione degli usi linguistici in relazione al variare dell'ambiente, delle abitudini, perfino delle condizioni abitative dei personaggi messi in scena. Se si segue la diversa connotazione linguistica dei testi, si osserva che lingua e dialetto si distribuiscono in modo variegato nelle opere di Eduardo De Filippo così come nella realtà cittadina. L'autore, in genere sempre attento alla verosimiglianza dei dialoghi messi in scena, segue attraverso le parole dei suoi personaggi una variabilità degli usi linguistici che da tempo caratterizza anche la vita quotidiana napoletana. Perciò sembra difficile condurre un discorso sulla "lingua di Eduardo», mentre appare plausibile parlare di usi linguistici che di volta in volta l'autore presta ai suoi personaggi, in rapporto al loro profilo sociale, all'ambiente in cui si muovono, ai contesti in cui avvengono i dialoghi.

Alla diversa collocazione, all'interno della topografia urbana, degli ambienti messi in scena sarà almeno in parte ascrivibile anche quel costante accostamento tra lingua e dialetto che spesso è stato sottolineato come una delle caratteristiche del teatro di Eduardo De Filippo. Non di rado, in verità, tale accostamento (con interferenza) tra le due varietà è stato interpretato come l'esito di una sorta di volontario "tradimento" del dialetto locale da parte dell'autore, talvolta sospettato di aver voluto consapevolmente annacquare il «puro" napoletano solo per rendere i suoi testi meno ostici alle orecchie di un pubblico non campano. In tale interpretazione è con ogni probabilità celato un giudizio fortemente limitativo sull'opera, o addirittura sull'autore, come forse sembra di indovinare dai non impossibili sottintesi di affermazioni come questa: ${ }^{2}$

A Viviani, d'altronde, Annibale Ruccello è vicino anche, se non soprattutto, sul versante della lingua, che nel suo caso è qualcosa di completamente diverso rispetto al compromesso linguistico tra italiano e napoletano adottato da Eduardo allo scopo di piacere, ossia di procurarsi il consenso anche lontano da Napoli.

Anche volendo accettare il principio, cosa in verità tutt'altro che scontata, che per un autore di teatro sia disdicevole l'intento di incontrare i favori di un pubblico non locale, si farebbe fatica ad accettare l'idea che la compresenza in scena di italiano e dialetto sia il prodotto di un compromesso (in quanto tale discutibile). Per di più è molto probabile che un'attenta analisi dei testi di Viviani e dello stesso Ruccello (autore anche di testi integralmente in italiano, e di altri in cui gli usi dialettali si alternano con quelli

1. Mi riferisco a Eduardo De FIlippo, Teatro. La cantata dei giorni pari, a cura di Nicola DE Blasi e Paola Quarenghi, Milano: Mondadori, 2000 e Eduardo De Filippo, Teatro. La cantata dei giorni dispari, tomo I, a cura di Nicola De BLASI e Paola QUARENGHI, Milano: Mondadori, 2005.

2. Enrico FIORE, Un po' voyeur, un po' poeta, in Annibale Ruccello, Teatro, Milano: Ubulibri, 2005, p. 9-17 (citazione da p. 15). 
italiani) ${ }^{3}$ porterebbe a osservare anche nelle opere di questi due drammaturghi una consistente dose di italiano, accostato e commisto al dialetto e forse perfino una compromissione vicendevole dell'uno e dell'altro. Sembra molto probabile in realtà che in tutti gli autori fin qui nominati la compresenza non rara di italiano e dialetto dipenda da una persistente intenzione di tener conto della realtà.

Per chi conosce dall'interno la realtà napoletana è facile notare come da una zona all'altra della città siano avvertibili sensibili differenze negli usi linguistici e nelle abitudini di vita, in una variazione percepibile all'interno della geografia sociale e linguistica cittadina: è intuitiva per esempio la constatazione che in talune zone il dialetto si parla più frequentemente che altrove. Tale constatazione si fonda con ogni probabilità sulla vicenda urbanistica della città di Napoli, che ha conosciuto nell'ultimo secolo un considerevole allargamento dei confini e una conseguente distribuzione degli abitanti, in parte diversa da quella, più uniforme, dei secoli precedenti.

La realtà urbana di Napoli in effetti si è molto modificata nell'arco di un secolo, nonostante una crescita demografica non eccezionale. Rispetto ai circa 995.171 abitanti attuali, ${ }^{4}$ la città nel 1927 contava circa 920.000 abitanti: una crescita di appena settantacinquemila in circa ottant'anni è davvero modesta, se si pensa che molti altri capoluoghi di regione nello stesso arco temporale hanno conosciuto incrementi ben più significativi.

A fronte di una stabilità demografica o di una crescita molto relativa, compensata in verità da uno straordinario incremento demografico della provincia, a Napoli si è invece molto modificata la consistenza territoriale della città (in relazione al totale delle aree edificate $)^{5}$ nonché la densità di popolazione. Fino al 1925 la città occupava una superficie di 65 Kmq., che in quell'anno è stata quasi raddoppiata, fino a $111 \mathrm{Kmq}$. con l'aggregazione di nove comuni limitrofi: S. Giovanni a Teduccio, Barra, Ponticelli, S. Pietro a Patierno, Secondigliano, Chiaiano e Uniti, Pianura, Soccavo. Già appartenevano all'area urbana i sobborghi di Posillipo, Fuorigrotta e Bagnoli, Miano, Piscinola e Marianella, che per la Guida del Touring del 1927 (fonte di questi dati) sono all'epoca ancora villaggi non ancora fittamente popolati. Prima di queste aggregazioni, Napoli contava 777.611 abitanti (nel 1921), ${ }^{6}$ diventati 920.000 per l'apporto dei comuni limitrofi; la densità abitativa corrispondeva nel 1921 a 12.000

3. Daria DI Bernardo, Dopo Eduardo: la lingua e la «nuova drammaturgia» di Annibale Ruccello, in Nicola DE BLASI e Tonia FIORINO (a cura di), Eduardo De Filippo scrittore, Napoli: Dante \& Descartes, 2004, p. 287-319.

4. Fonte è il Calendario Atlante 2006, Torino: De Agostini, 2005. Secondo i dati del censimento, invece, nel 1991 risiedevano in città 1.067.365 abitanti (Dati Istat, 1995).

5. Sottolinea Giuseppe Galasso (a cura di), Napoli, Bari - Roma: Laterza, 1987, p. XXXV che la principale attività economica della città nel corso del Novecento è stata un'edilizia anche "tracotantemente speculativa".

6. Per valutare quanto sia ridotto il saldo positivo registrato in quasi ottant'anni, è il caso di notare che in appena vent'anni, tra 1901-1921, la popolazione cittadina si accrebbe di oltre duecentomila unità, da circa 563.000 a circa 772.000 . 
abitanti per Kmq., assiepati in tredici quartieri (in alcuni dei quali la densità oltrepassava di molto i 20.000 abitanti): S. Ferdinando, Chiaia, Montecalvario, Avvocata, S. Giuseppe, S. Lorenzo, Porto, Stella, S. Carlo all'Arena, Vicaria, Mercato, Pendino, Vomero, oltre che nei sobborghi.

L'elevata densità abitativa è stata un requisito costante della città, che già a fine ' 400 contava circa centomila abitanti, collocati però in un'area più $o$ meno corrispondente ai soli quartieri di San Giuseppe, San Lorenzo, Porto, Vicaria, Mercato, Pendino. ${ }^{7}$

In rapporto alla «piccola» Napoli dei primi anni Venti, si trovavano pertanto in estrema periferia, allo stato di sobborghi rurali, quelle zone poi fittamente urbanizzate nei decenni successivi, da Posillipo a Bagnoli, da Fuorigrotta al Vomero, mentre per altre non esisteva nemmeno il toponimo poi invalso nella toponomastica ufficiale (p. es. Cavalleggeri Aosta) o «spontanea», ma poi in qualche modo ratificata (p. es. Rione Alto). ${ }^{8}$ La città si distendeva in sostanza in un'area compresa tra la zona della Ferrovia e Mergellina, spingendosi all'interno fino al quasi inabitato Vomero (via Aniello Falcone in via di costruzione era considerata come una circonvallazione collinare) e ai piedi di Capodimonte: questo era anche il perimetro che delimitava l'area dialettale napoletana in senso stretto, dal momento che tutto ciò che si trovava al di fuori era percepito come realtà diversa e ben distinguibile da Napoli.

Tale prima constatazione comporta subito una riflessione sulla situazione linguistica presente: oggi risulta a prima vista singolare che un grosso centro come Pozzuoli, pur tanto vicino al capoluogo, abbia un dialetto molto caratterizzato (con i suoi tipici dittonghi). Pur senza sottovalutare altre ragioni di tali differenze (quali per esempio l'affermazione di una propria identità linguistica), si noti che fino ai primi decenni del Novecento Pozzuoli era solo relativamente vicina a Napoli, in quanto ne era separata da alcune alture, oltre che $\mathrm{da}$ aree rurali che certo poco potevano interferire dal punto di vista dialettale con la realtà puteolana (a cui anzi — si pensi ad Agnano o Bagnoli- saranno state piuttosto affini). Abituati come siamo a considerare, con un approssimativo appiattimento sul presente, l'intera fascia costiera nei dintorni di Napoli come un unico lungo insediamento abitativo, forse stentiamo ad assuefarci all'idea che nel territorio cittadino vi fossero, settanta o ottanta anni fa, ampi spazi ancora non urbanizzati o sobborghi ben separati dal resto della città.

7. Nei quartieri della «vecchia Napoli» (San Ferdinando Chiaia, San Giuseppe, Montecalvario, Avvocata, Stella, San Carlo all'Arena, Vicaria, San Lorenzo, Mercato, Pendino, Porto), abitavano, nel 1921, 770.000 persone (o poco meno se si pensa ai non numerosi abitanti, per esempio, di Posillipo o del Vomero). In quest'area centrale, secondo gli ultimi censimenti (1995 e 2001) la popolazione nel corso del secolo si è più che dimezzata, mentre si sono affollati i quartieri più nuovi (Vomero, Arenella, Posillipo, Poggioreale, Zona industriale, Bagnoli, Fuorigrotta, Soccavo, Pianura, Chiaiano, Piscinola, Miano, Secondigliano, Scampia, San Pietro a Patierno, Ponticelli, Barra, San Giovanni). Per gli aspetti storico-linguistici a cui si fa cenno qui rinvio a Nicola DE BLASI, «Per la storia contemporanea del dialetto nella città di Napoli», Lingua e stile, n. 37, giugno 2002, p. 123-157.

8. Rione alto è il nome di una fermata della Metropolitana collinare. 
Fino alla fine dell'Ottocento, dunque, abbiamo una grande capitale di respiro europeo che in un certo senso è una città piccola, in cui gli abitanti vivono tutti in un'area relativamente ristretta: anche ceti tra loro diversi convivono, per così dire, a stretto contatto di gomito, anche se si diversificano quanto a condizioni sociali e livello culturale. Com'è noto, in una città più compatta e di minore ampiezza, tutti i quartieri erano in ugual misura condizionati dalla struttura del tipico palazzo napoletano in cui ceti diversi convivevano (anche se in piani diversi), accomunati anche dal dialetto usato. Si legga al riguardo quanto osserva lo storico Giuseppe Galasso in riferimento all'età moderna: ${ }^{9}$

L'uso stesso del dialetto locale non è una esclusività popolare, poiché la relativa tradizione ha tanto componenti popolane quanto componenti «borghesi», e perfino aristocratiche o para-aristocratiche (si ricordi sempre la fondamentale sociologia del Popolo napoletano tracciata dal Capaccio). ${ }^{10}$ L'uso, anzi, della «lingua napoletana» si sarebbe mantenuto indefinitamente, ben al di là del periodo di cui si parla, come un segno di identità sociale e culturale a valenza assolutamente interclassistica, in conformità ad altri caratteri della società napoletana (basti pensare alla realtà del palazzo napoletano e alla sua singolarità di vero microcosmo sociale per la promiscuità di classi e di ceti che esso rispecchia nella sua fisionomia residenziale).

Abitando all'interno degli stessi quartieri, spesso perfino nello stesso palazzo «microcosmo", popolani e borghesi, artigiani e uomini di cultura, nobili e plebei, condividevano insomma lo stesso universo comunicativo e interagivano gli uni con gli altri, trovando nel dialetto — semmai usato in modi diversi da ceto a ceto- uno strumento di comunicazione valido e pronto all'uso.

Dalla fine dell'Ottocento nella città che si andava allargando si sono delineati quartieri con diversificate vocazioni abitative: dal centro antico alcuni abitanti si sono allontanati verso alcuni quartieri, altri si sono diretti verso altre zone, mentre proseguiva, sia pure a rilento, il persistente afflusso di abitanti dalle province che da sempre è stata una caratteristica costante nella storia urbana: gli irpini, i calabresi, i lucani, i sanniti, i cilentani, gli abruzzesi che giungevano in città, nel Novecento come in passato, importavano a Napoli i loro dialetti di origine, ma qui incontravano sia il dialetto locale, sia l'italiano. Non si dimentichi infatti che da sempre l'immigrazione che dalle pro-

9. Giuseppe GaLASSO, "Da "Napoli gentile" a "Napoli fedelissima”", Annali dell'Istituto Suor Orsola Benincasa, n. 1, 1996, p. 47-121.

10. Il riferimento è al secentesco descrittore di Napoli, Giulio Cesare Capaccio, che nell'opera Il Forastiero enumera i ceti napoletani: «Tutti questi distinguo in nobili, popolani e plebei. I primi, di Piazza, e fuor di Piazza. I secondi, in megliori, e di mezzana conditione. I terzi in gente bassa" (Giulio Cesare CAPACCIO, Il forastiere, Napoli: Luca Torre, 1989, tre volumi, p. 468); il Capaccio elenca tre strati di popolo (quello dei gentiluomini, dei tribunali e dei mercanti: ibid., p. 525) e tre di plebe (che vive civilmente, che vive poco civilmente, che vive in bassezza). 
vince si è diretta verso Napoli è stata in larga misura un'immigrazione intellettualmente qualificata, che veniva a Napoli proprio per intraprendere o proseguire gli studi (si pensi per esempio ai casi celeberrimi di Francesco De Sanctis, Francesco Torraca, Benedetto Croce). Per costoro, dunque, Napoli è stata la città dell'italiano prima e più che la città del napoletano: questa precisazione dà forse una prima idea di come possa essere solo parzialmente fondata l'idea che a Napoli tutti conoscano o parlino correntemente il napoletano. A tutti è noto come non sia per nulla una cosa scontata che una cittadino residente a Napoli abbia tutti e quattro i nonni di origine napoletana; è intuibile quali siano le implicazioni di una situazione del genere, se si pensa alle biografie linguistiche dei diversi parlanti. Vale a dire che se oggi qualcuno non parla il napoletano è molto probabile che ciò non dipenda da un rifiuto del dialetto, ma semplicemente da oggettive ragioni anagrafiche risalenti alle famiglie d'origine. D'altra parte, in questa stessa città, il dialetto è tuttora lingua primaria e prevalente di una buona parte della popolazione, che pure da parte sua non intende in alcun modo marcare un rifiuto o una distanza nei confronti dell'italiano. Si può quindi affermare che una parte della città si è orientata preferibilmente (anche per circostanze legate alla storia di ciascuna famiglia) verso l'italiano, mentre per un'altra parte il dialetto è tuttora lingua prevalente. ${ }^{11}$

Nella realtà cittadina, tanto differenziata al suo interno, immettono direttamente le commedie di Eduardo De Filippo, che da grande autore di teatro percepisce e coglie le variazioni interne alla città. Ogni volta che deve dar vita a un personaggio, l'autore è quindi bene attento a calarlo in un contesto linguisticamente e socialmente credibile.

Pertanto non ci stupiamo che in una commedia ambientata in un basso, come Napoli milionaria!, il dialetto abbia uno spazio considerevole e ceda il posto all'italiano solo quando il protagonista Gennaro Jovine si sbilancia in considerazioni sugli oscuri interessi economici di coloro che regolano il calmiere, incoraggiando di fatto la borsa nera.

In questo ambiente cittadino dove italiano e dialetto convivono da sempre a stretto contatto, i parlanti si trovano continuamente davanti a una scelta sulla lingua da usare: la vicinanza tra i due codici linguistici fa sì che tali scelte non siano inconsapevoli. I parlanti si rendono conto delle circostanze in cui l'italiano è più adeguato del napoletano e viceversa. Per esempio Filumena Marturano è una commedia in cui si determina una sorta di confronto esplicito tra le due lingue, tanto che ne deriva anche una esplicita indicazione metalinguistica. Filumena infatti in ben due occasioni nomina e definisce la sua scelta a favore del napoletano: Filumena, figlia del popolo, con un'infanzia e adolescenza di sofferenza, in una delle scene chiave della commedia, quando l'avvocato del marito cerca di spiegarle che il matrimonio non è valido perché

11. Per questi aspetti rinvio a Carla Marcato e Nicola De Blasi, «Lo spazio del dialetto in città. Il napoletano a Napoli», in Gianna Marcato (a cura di), Dialetto in città, Atti del Convegno di Sappada (1-4 luglio 2004), Padova: Unipress, 2005, p. 115-121. 
lei non è mai stata in punto di morte, dichiara la sua adesione istintiva al dialetto. Quando l'avvocato le legge gli articoli del codice, la donna dice di non capire, manifesta il suo disagio perché con la sua istintività non riesce a comprendere come il codice possa essere contro i suoi diritti naturali. Da ciò nasce un'esplicita distanza linguistica: «Avvoca', spiegateve â napulitana» dice Filumena. In questo momento le due lingue arrivano in una condizione di frizione. Filumena non capisce quell'italiano delle leggi, fredde e prive di pietas. La donna, che pure a volte parla in italiano nella commedia, in quel contesto lo rifiuta.

In un altro passaggio di questo stesso dialogo la protagonista, mentre parla del vicolo e del basso dove abitava da ragazza, traduce: «Addó nun ce sta luce manco a mieziuorno... Io parlo napoletano, scusate... Dove non c'è luce nemmeno a mezzogiorno..... ${ }^{12}$ Qui le scuse nascono non dal timore di non essere compresa dagli interlocutori, ma dal fatto che italiano e napoletano si collocano per Filumena su due piani diversi: l'italiano è la lingua delle leggi, mentre il napoletano, per lei lingua materna, è il codice dell'espressione spontanea e affettiva. Dal suo basso di vico San Liborio, dunque, Filumena si trova a dialogare con un avvocato, nell'agiato interno borghese dell'appartamento di don Domenico Soriano, ricco proprietario di alcune pasticcerie. In quello stesso appartamento (che l'autore avrà immaginato in un palazzo simile a quello di via San Potito, di fronte al Museo Nazionale, dove è ambientata la storia nella versione cinematografica del 1950), il napoletano convive con l'italiano, ma si scontra anche con l'inglese dell'ultima "conquista» di don Domenico, la donna alla quale con intenzione aggressiva Filumena si rivolge esclusivamente in dialetto, costringendo la fedele Rosalia al ruolo di traduttrice oltre che di passaparola.

In altre commedie, anche se permane un certo uso del dialetto, l'italiano è di gran lunga prevalente: è il caso del testo Le bugie con le gambe lunghe, che è ambientata significativamente non nel centro di Napoli ma in un palazzo borghese del Vasto, uno dei nuovi quartieri nati nel corso del '900. L'autore sottolinea dunque che la vicenda accade in un rione della città di edificazione risalente a pochi decenni prima (e pochi decenni sono davvero pochi in una città $\mathrm{i}$ cui quartieri più antichi sono ininterrottamente abitati da oltre duemila anni). L'immenso fabbricato in stile Novecento è una costruzione edificata all'inizio del secolo ventesimo, in uno dei nuovi quartieri, in cui la popolazione proveniente in parte dal centro antico, in parte dalla provincia, cominciò a distribuirsi in collocazioni differenziate rispetto alle diverse condizioni sociali. Siamo insomma lontani dalla città popolare di Forcella, del Cavone, del Pallonetto, cioè dei quartieri antichi di cui si parla in Napoli milionaria!, quelli in cui il popolo viveva a stretto contatto con gli impiegati e con i possidenti o i nobili. Siamo anche lontani sia dall'enorme palazzo nobiliare in cui si installa Pasquale Lojacono in Questi fantasmi!, sia dal vicolo San Liborio dove nasceva Filumena Marturano. 
Il quartiere del Vasto, che a Napoli rappresenta uno dei nuovi insediamenti sorti tra l'epoca dell'Unità e i decenni tra le due guerre; è appunto uno dei quartieri di prevalente qualificazione borghese che, nella percezione soggettiva degli abitanti, ma anche oggettivamente, presenta caratteri socio-ambientali piuttosto diversi rispetto ai quartieri più antichi e a quelli popolari più recenti. Non è quindi improbabile che l'autore voglia sottolineare che siamo in quartiere moderno, con palazzi alti, che può trovarsi a Napoli come in qualsiasi altra città italiana.

In questo contesto, l'appartamento di Libero e Costanza, per quanto umile, si qualifica subito per le sue differenze rispetto alle altre case che abbiamo visitato finora nell'opera di Eduardo De Filippo. All'inizio siamo introdotti in un appartamento al sesto piano di un caseggiato servito da ascensore: per altezza e per stile di vita siamo in ambienti diversi da quelli in cui maturano altre storie eduardiane. Qui manca quell'apertura costante verso l'esterno e verso i vicini che si riscontra per esempio nell'habitat della famiglia Jovine in Napoli milionaria!: la vita di famiglie diverse all'interno dello stesso palazzo-alveare scorre su binari paralleli che possono non incontrarsi, tanto che capita di non conoscere i vicini o accade di poter fingere di non conoscerli. Questo clima di potenziale isolamento che può mutarsi in totale estraneità favorisce la consuetudine della finzione ipocrita o delle bugie compiacenti, che nel piccolo mondo del vicolo, dove tutti si conoscono da sempre, non avrebbero invece vita facile né "gambe lunghe».

In questo ambiente, che gioca un ruolo importante nel rendere in qualche modo plausibile tutto l'intreccio, è comprensibile che si usi l'italiano più del dialetto, anche perché in casa del protagonista si vedono "libri curati e ben disposti un po' dappertutto». I libri (tanto più se numerosi e sparsi un po' dappertutto) rappresentano un indizio culturale di forte rilievo, poiché denotano uno stretto e continuo rapporto con la scrittura e con la lingua italiana. Del resto Libero Incoronato solo attraverso la scrittura e i libri può seguire le vicende storico-politiche che si traducono poi in serie filateliche. Con i libri «curati e ben disposti», anche il resto dell'arredo è «spolverato e lucido», pur rivelando senza equivoci una povertà dignitosa, ma tangibile: i mobili sono vecchi, al pari del tappeto sul tavolo, la tovaglia è rattoppata, le stoviglie sono spaiate, i quadri sono insignificanti, il lampadario ha una sola lampada accesa. Questi oggetti di uso quotidiano logori e in un certo senso provati anch'essi dagli stenti, come la camicia rattoppata del secondo atto, sono altrettanti segnali di realismo, e confermano che Libero e la sorella, nelle loro condizioni, non possono cullare alcuna ipocrisia illusoria, ma devono rimanere con i piedi per terra, secondo la netta dichiarazione di Libero ("Qui viviamo di realtà ora per ora, minuto per minuto»). Con piccoli inserti in dialetto, Libero Incoronato sottolinea l'inutilità di certe preoccupazioni perbenistiche della sorella che non vorrebbe svelare le proprie condizioni economiche non agiate: $^{13}$ 
Il vino l'ho comprato, ed ho comprato pure il formaggio. Cinquanta grammi. (A Costanza) E tu, che hai preparato? (Costanza non risponde). Costa, che hai preparato per cena? (Costanza, infastidita, gli fa dei gesti come per fargli intendere che in presenza di Graziella non vuole parlare delle loro miserie). Costa, ma tu fai sul serio? Ma perché 'a signurina nun 'o ssape ca stammo nguaiate e ca Dio 'o ssape comme campammo? Secondo te, se non mi dici che cosa hai preparato per cena, 'a signurina Graziella se ne va convinta ca stasera mangiammo dentice e aragosta.

In un ambiente diverso è informatissimo su tutto il vicinato il portiere dell'antico e storico palazzo del centro in cui è collocato l'ambiente di Questi fantasmi!; nel presentare il professore che abita nel palazzo di fronte, il portiere è prodigo di notizie minute che tracciano un sintetico profilo biografico, anagrafico, economico e perfino sanitario di questo personaggio: ${ }^{14}$

Il professor Santanna. Fa lezione a 'e studiente; tiene la scuola in casa. È vedovo e s'è nzurato n'ata vota. Perdette na sora a 'o terremoto 'e Messina. Tene na piccola proprietà â parte 'e Salierno. $\mathrm{Nu}$ frate in America 'a trentadue anni. Tene 'o diabete...

In Questi fantasmi! con il suo bilinguismo funzionale il portiere è la figura chiave che garantisce la sutura tra il mondo esterno e l'interno del palazzo. Nel rivolgersi al professore che abita di fronte, il protagonista Pasquale Lojacono usa senza incertezze l'italiano, che forse è suggerito anche dallo status culturale dell'interlocutore. Ecco un passaggio del celebre dialogo (/monologo) del caffe, che è interamente in italiano: ${ }^{15}$

A noialtri napoletani, toglieteci questo poco di sfogo fuori al balcone... Io, per esempio, a tutto rinuncierei, tranne a questa tazzina di caffe, presa tranquillamente qua, fuori al balcone, dopo quell'oretta di sonno che uno si è fatta dopo mangiato. E me la devo fare io stesso, con le mie mani. Questa è una macchinetta per quattro tazze, ma se ne possono ricavare pure sei, e se le tazze sono piccole pure otto... per gli amici... il caffe costa così caro...

D'altra parte lo stesso Pasquale, per contendere con il portiere e per far fronte ai suoi tentativi di sottrargli oggetti o denaro, non esita a usare il dialetto. Il portiere da parte sua, proprio per la sua posizione di confine tra dentro e fuori, tra italiano e dialetto, cade a volte in quell'inconsapevole interferenza che caratterizza il cosiddetto italiano popolare. In Questi fantasmi!, come sempre, all'italiano si affianca il dialetto, che però rimane confinato in ambiti ben definiti: da acuto osservatore della realtà, Eduardo, sia nel manoscritto sia nelle edizioni in volume, presenta un universo cittadino in cui, al di là delle semplificazioni banalizzanti, non tutti parlano allo stesso modo. Nel piccolo mondo 
del palazzo dello spagnolo Los De' Rios, ideale proiezione teatrale di vari possibili palazzi, immensi e principeschi, davvero presenti a Napoli (dal palazzo Tarsia fino al cosiddetto palazzo dello Spagnolo nel borgo dei Vergini), l'apertura verso il dialetto è rappresentata da Raffaele, che a volte involontariamente lascia affiorare il dialetto anche quando vorrebbe parlare in italiano (come in solidarietà per solitudine o nella frase «Per quanto uno può essere a conoscenza dei scherzi ca fanno questi fantàseme...»).

Senza alcuna mediazione è invece direttamente a contatto con la strada la vita che si svolge in un basso: perciò nel basso di Napoli milionaria!, insieme con i neologismi italiani di attualità, irrompono parole di origine americana debitamente adeguate al napoletano: la figlia del protagonista si fidanza con un americano, e, frequentando anche i suoi amici, lei e le sue compagne cominciano a usare alcune parole inglesi. Come per esempio in queste battute pronunciate da Teresa, un'amica della figlia del protagonista: ${ }^{16}$

È un'altra mendalità: sono più sciolti, più abbonati... Chillo, Gion, faceva ammore primma cu me. Po' cunuscette a Maria Rosaria, e dicette ca lle piaceva cchiù assaie. M' 'o ddicette nfaccia: «Tua frenda più nais!» Io rispunnette: «Okei!»'A sera purtaie n'atu frend suo ca sùbbeto s'annammuraie 'e me e a me me piaceva più di lui e ce mettèttemo d'accordo. Io poi le dicette: «Ci ho na frenda mia, che sarebbe Margherita, non ci hai un frendo tuo?» Isso 'o purtaie e accussì avimmo fatto tre freind e tre freende.

Di tutt'altro genere è il contesto abitativo in cui si svolge La paura numero uno. Qui il protagonista Matteo Generoso è un agiato borghese che a quanto pare vive di rendita e solo per passatempo ha assunto la carica di amministratore di condominio di un palazzo i cui balconi si aprono sulla collina di Posillipo. Ci siamo dunque allontanati sia da vico san Liborio, sia dal basso di Napoli milionaria!, sia dal palazzo del centro antico, sia dalla semi-periferia orientale: il palazzo panoramico è in un quartiere residenziale collinare ed è abitato direttamente dai proprietari, che curano con molta attenzione i propri interessi. In questo ambiente c'è spazio per una vicen$\mathrm{da}$ che, in ossequio ai tempi di incipiente boom economico (qui, per esempio, appare per la prima volta in un testo letterario la parola lambretta), è segnata dalle nevrosi e dalle fobie di alcuni personaggi. In un palazzo di questo genere, tra vasi fioriti, giardini e vista panoramica, l'italiano è la lingua abituale, anche se il dialetto non scompare mai del tutto. Sembra tuttavia che il dialetto sia come citato e ripreso come una lingua usata da altri, come il mezzo espressivo del mondo di fuori. A questo proposito è significativo che Matteo Generoso, nel manifestare i suoi timori di una guerra imminente, riferisca di un incontro con veloce scambio di battute avvenuto in via Toledo: ${ }^{17}$ 
Virgi', ci siamo. Ho visto una ragazza a Toledo, non c'era male... che mordeva, più che mangiare, un cannolo alla siciliana, ridendo con una sua compagna, che le diceva: «Damménne nu poco!...» Dal tono, ho capito che voleva dire: «Forse è l'ultimo cannuolo che ci mangiamo insieme: è meglio ca ce 'o spartimmo». Mi sono avvicinato con molta delicatezza, e ho detto: «Non c’è bisogno di dividere quello. Ve lo compro io un altro cannuolo... Venite!» Una delle due ha risposto con una sovreccitazione intollerante, dovuta al clima in cui viviamo: «'A tiene na sora bona? Ce l'accatte a soreta 'o cannuolo. Cammina, Cuncetti'!» Hanno affrettato il passo, e le ho viste scomparire in mezzo alla folla...».

Dal punto di vista di Matteo Generoso, il dolce che le due ragazze mangiano è un cannolo, ma dal discorso riportato si comprende per le due ragazze quello stesso dolce è un cannuolo: in questa minima differenza tra cannolo e cannuolo c'è in un certo senso una distanza linguistica e ambientale tra chi parla in italiano anche in famiglia (pur comprendendo e adottando talvolta il napoletano) e chi spontaneamente parla soltanto in dialetto.

Nei nuovi quartieri di epoca postunitaria, nei ceti dotati di taluni requisiti socio-culturali il dialetto sembra dunque perdere la sua funzione di lingua primaria della conversazione spontanea: a ciò potrebbe collegarsi, apparentemente, un suo irrimediabile declino. L'autore Eduardo De Filippo si accorge però che tale declino nella realtà è tutt'altro che scontato: il napoletano infatti dispone di una risorsa aggiuntiva. Anche chi non parla questo dialetto spesso lo apprezza e lo valorizza, mostrando nei suoi confronti un atteggiamento positivo: ciò accade nella realtà (si pensi a quanti sono, a Napoli e nel mondo, gli appassionati estimatori della canzone, della poesia, del teatro, del cinema in napoletano), come accade del resto nelle stesse commedie di Eduardo.

Di questo atteggiamento positivo verso il dialetto, anche in un contesto borghese e da parte di un personaggio che parla in italiano, si trova traccia nella commedia Mia famiglia, dove un personaggio che si chiama Guidone si diffonde in un'esplicita esaltazione della forza espressiva del dialetto: ${ }^{18}$

Quello che ho detto io. Beppe, senti a me, frocoleaténne. (Ripensando all'espressione dialettale che gli è venuta alle labbra, socchiude gli occhi estasiato, ripronunciando la parola per assaporarne tutto il gusto che gliene viene, ogni qual volta può dimostrare agli altri quale raffinato conoscitore egli sia di battute, frasi e motti partenopei) Frocoleaténne! Sentite, io credo che non ci sia al mondo nessun altro dialetto capace di poter esprimere qualunque sensazione e stato d'animo: frocoleaténne!... come se il mondo si frantumasse in minutissime scaglie di mica... Come se un'enorme torta millefoglie sparpagliasse felice le sue squame profumate alla vaniglia sulla tela di Penelope... La parola ha in se stessa una miracolosa scala musicale, ricca di semitoni e di bemolli. Frocoleaténne! (Traducendo in lingua) Lascia che il mondo caschi, non te ne dare per inteso... (E conclude convinto) Io so' pazzo p' 'o dialetto nostro. 
Nelle commedie di Eduardo De Filippo, in conclusione, troviamo indizi di diversi atteggiamenti verso il dialetto: dal dialetto usato come lingua spontanea e affettiva all'interferenza involontaria con l'italiano e l'inglese, dal dialetto evitato al dialetto osservato dall'esterno, fino al dialetto evocato e assaporato. In questa variegata gamma di punti di vista troviamo dunque testimoniate alcune delle tante possibili percezioni e valutazioni del dialetto che costantemente è possibile tuttora osservare in una città dagli usi linguistici tutt'altro che uniformi. 